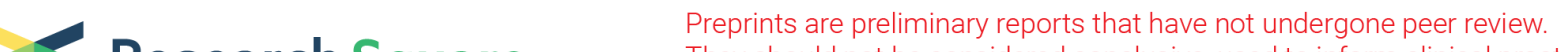 Research Square They should not be considered conclusive, used to inform clinical practice, or referenced by the media as validated information.
}

\section{Evaluate the impact of maternal smoking during pregnancy on sleep duration in female offspring}

Jingxi Zhang

Xi'an Jiaotong University https://orcid.org/0000-0001-5600-9897

Peilin Meng

Xi'an Jiaotong University

Chuyu Pan

Xi'an Jiaotong University

Jing Ye

Xi'an Jiaotong University

Huijie Zhang

Xi'an Jiaotong University

Chun'e Li

Xi'an Jiaotong University

Yujing Chen

Xi'an Jiaotong University

Zhen Zhang

Xi'an Jiaotong University

Shiqiang Cheng

Xi'an Jiaotong University

Li Liu

Xi'an Jiaotong University

Xuena Yang

Xi'an Jiaotong University

Yao Yao

Xi'an Jiaotong University

Yumeng Jia

Xi'an Jiaotong University

Feng Zhang ( $\nabla$ fzhxjtu@mail.xjtu.edu.cn )

Xi'an Jiaotong University Health Science Center

Research article 
Keywords: Maternal smoking during pregnancy, Gene, Sleep duration, Genome-wide gene-environment interaction study

Posted Date: October 1st, 2021

DOI: https://doi.org/10.21203/rs.3.rs-940203/v1

License: (c) (i) This work is licensed under a Creative Commons Attribution 4.0 International License. Read Full License 


\section{Abstract \\ Background}

Sleep is a fundamental state of decreased activity and alertness conserved in human life. Maternal smoking during pregnancy may affect sleep duration female offspring, however the exact connection and mechanism still remain unclear.

\section{Methods}

Based on the UK Biobank data, sleep duration was categorized as short sleep ( $<7 \mathrm{~h} /$ day) and long sleep ( $\geq 9 \mathrm{~h}$ /day). Logistic regression analysis was established to evaluate the potential impact of maternal smoking during pregnancy on the short sleep and long sleep of female offspring. Subsequently, genomewide gene-environment interaction study (GWGEIS) of sleep duration was performed using maternal smoking during pregnancy as an environmental factor.

\section{Results}

We found that maternal smoking during pregnancy was related to short sleep and long sleep duration of female offspring $(P<0.001)$. GWGEIS analysis detected a significant association at ANKLE2 (rs78408032, $P=1.33 \times 10^{-8}$ ) and a suggestive association at MYT1L (rs76688230, $\left.P=8.85 \times 10^{-7}\right)$ in female subjects.

\section{Conclusion}

Our study results suggested a significant effect of maternal smoking during pregnancy on short sleep of female offspring.

\section{Introduction}

Sleep occupies a third of the people's lives. The quality and duration of sleep could affect both psychological and physical health of children and adults, especially in cognition and execution. Proper and highly quality sleep enables person having sufficient ability to cope with the problems in life and work. Sleep disturbance includes short and long sleep duration, insomnia, signs of daytime dysfunction and difficulty awakening in the morning, which are recognized as the risk factor of human health. For example, long-term sleep duration is associated with increasing the risk of stroke and breast cancer [1, 2], and inadequate sleep duration is considered as the risk factor of cognition, memory and neurologic diseases, which decreases vigour and heightens fatigue $[3,4]$. 
Sleep problems are generally linked to both environmental and genetic factors. Environmental factors involve alcohol, skin warming and social issues. For instance, previous study has shown that proper skin temperature facilitates the onset of sleep [5]. It has been commonly known that genetic factors contributed greatly to the regulation of sleep duration. Y Dauvilliers et al and M Partinen et al. have proved that sleep length can be heritable [6, 7]. For example, it was suggested that sleep problems were related to $F X R 1$ and $D C E 2$ gene mutation $[8,9]$.

Cigarette smoke contains approximately 4700 substances, many of which are carcinogenic. Cigarettes are one of the most important causes of lung cancer, and the cancer for recent research have established a causal related to tobacco smoking are oral, nasal cavities, larynx, liver, oesophagus, pharynx, stomach, kidney, urinary tract and cervix [10]. As the number 1 and the number 1 preventable cause of death, cigarette smoking contributes to 6 million deaths every year, and secondhand smoke is responsible for the death of more than 600,000 individuals worldwide [11]. Unfortunately, pregnant women are often exposed to tobacco in their daily lives, either passively or actively.

Recently, it has been commonly known that maternal smoking during pregnancy (MSDP) takes considerable risk for baby. Compelling correlative evidence claims that ingredients in cigarette smoke could greatly harm the newborn via placenta [12]. Some studies suggested that maternal smoking during pregnancy may change the glutamatergic system in the hippocampus, and increase the risk of abnormal neurobehavioral outcomes in offspring [13]. And, maternal smoking is associated with altered total brain volumes and cortical gray matter volumes, which indicated that exposure to cigarette smoke environment in utero may impact the brain function and result in neurodevelopmental abnormalities [14]. Sleep disturbance is associated with exposure to high levels of tobacco smoking [15]. Prenatal smoking exposure could be concerned with altering neurodevelopment, which affects offspring sleep [16]. It's reported that maternal smoking during pregnancy affects offspring significantly greater in female than in males [17], therefore, we would like to pay more attention to sex-specific risk of maternal smoking during pregnancy on the sleep duration in female offspring.

In the study, we performed observational analyses and GWGEIS to investigate the association between maternal smoking during pregnancy and sleep duration in female offspring, based on UK Biobank samples. We aimed to explore the relationship between maternal smoking during pregnancy and sleep duration, and detect novel candidate genes that affect sleep duration by interacting with maternal smoking during pregnancy in females.

\section{Methods And Materials}

\section{UK Biobank}

UK Biobank is a large scale prospective, this cohort contained about 500,000 subjects aged between 37 and 76 years ( $99.5 \%$ were aged $40-69$ years). All involved subjects provided a sequence of information on demographics, health status, and lifestyle get through questionnaires and interviews [18]. The UK 
Biobank has obtained informed consent of all participants and ethical approval from the Northwest MultiCenter Research Ethics Committee. Obtain the informed consent of all participants.

\section{Phenotype definition}

Sleep duration was recorded as number of reported hours by asking 'About how many hours sleep do you get in every $24 \mathrm{~h}$ ? (include naps). And then we categorized the sleep duration as short sleep ( $<7 \mathrm{~h} /$ day), normal (7-8h/day), and long ( $\geq 9 \mathrm{~h} /$ day) consistent with previous studies [19]. Smoking phenotype is defined as "Did your mother smoke regularly around the time when you were born?". If the answer is yes, it is defined as smoking, and individuals were classed as never smoke if answered to question "No". Individuals who answered to either question "Do not know", "Prefer not to answer" were excluded in this study (UK Biobank code: 1787). The age and gender data of study subjects was derived from the baseline characteristics of the UK Biobank.

\section{Genotyping, imputation and quality control}

In UK Biobank Affymetrix UK BiLEVE Axiom and Affymetrix UK Biobank Axiom was used for SNP genotyping. Sample-based and label-based quality control were performed. Imputation was conduction by IMPUTE4. Principal components (PC) were computed to account for population structure. We restricted study subjects to the 'White British' group based on self-reported ethnic background (UK Biobank data field: 21000). Genetically related individuals were excluded according to the kinship coefficient calculated by the KING software. Details about imputation, genotype and quality control are available in previous studies [20].

\section{Observational analyses}

Logistic regression analysis was used to evaluate the relationship between short/long sleep duration and maternal smoking during pregnancy in female offspring. Short sleep duration, long sleep duration and maternal smoking during pregnancy was set as binary variable. 10 principle components, age, daily frequency of smoking were used as covariates in the logistic regression models.

\section{Genome-wide gene-environmental interaction study (GWGEIS) analyses}

Maternal smoking during pregnancy was regarded as an environmental factor, we performed GWGEIS of female short/long sleep duration by PLINK2.0 [21]. This method considered the impact of SNP environment interaction from a regression model [22]. The GWAS $P<5.00 \times 10^{-8}$ and $P<1.00 \times 10^{-6}$ were used as significant and suggestive threshold, respectively. For quality control, we excluded the SNPs with low call rates $(<0.90)$, low Hardy-Weinberg equilibrium exact test $P$ values $(<0.001)$, or low minor allele frequencies (MAFs<0.01) in this study.

\section{Results}




\section{Basic characteristics of study subjects}

Our study includes a total of 175135 female samples. Smoking sample contains a total of 52599 participants and the mean (standard deviation) age was 55.96 (7.59). Never smoke sample contains a total of 122536 participants and the mean (standard deviation) age was 56.86 (7.99). The basic characteristics of female offspring sleep duration in smoking and never smoke during pregnancy were presented in Table 1.

Table 1

The sleep duration of female offspring with and without maternal smoking during pregnancy

\begin{tabular}{|lll|}
\hline & Maternal smoking & No smoking \\
\hline $\mathbf{N}$ & 52599 & 122536 \\
\hline Age(SD) & $55.96(7.59)$ & $56.86(7.99)$ \\
\hline Short sleep(\%) & $4372(11.20)$ & $9608(10.10)$ \\
\hline Long sleep(\%) & $13334(27.80)$ & $26946(24.00)$ \\
\hline
\end{tabular}

\section{Observational Analyses Results}

In the logistic regression analysis, short-term sleep $\left(O R=1.23[1.20,1.26], P<2.00 \times 10^{-16}\right)$ was related to maternal smoking during pregnancy in females. Logistic regression analysis showed that long-term sleep $\left(\mathrm{OR}=1.11[1.06,1.16], P=3.34 \times 10^{-10}\right)$ was related to maternal smoking during pregnancy in females.

\section{Genome-wide Environmental Interaction Analyses}

For short sleep duration, GWGEIS identified a significant association at ANKLE2 (rs78408032, $P=$ $\left.1.33 \times 10^{-8}\right)$ in female offspring. In addition, we also observed several candidate loci with suggestive association for short sleep duration, such as rs76688230 $\left(P=8.85 \times 10^{-7}\right)$ of MYT1L gene on chromosome two, rs212421 $\left(P=1.30 \times 10^{-6}\right)$ of ATXNTL1 gene on chromosome seven, rs72767679 $(P=$ $\left.1.39 \times 10^{-6}\right)$ of CAMK1D gene on chromosome ten, and $\operatorname{rs} 10206895\left(P=1.78 \times 10^{-6}\right)$ of SERPINE2 gene on chromosome two. For long sleep duration, we identified candidate loci with suggestive association signals, for instance rs78942972 $\left(P=4.77 \times 10^{-7}\right)$ and rs61925708 $\left(P=1.78 \times 10^{-6}\right)$ of ANO6 gene on chromosome twelve. Additional results were listed in Table 2 and Table S1. The circular Manhattan plots for long and short sleep duration were displayed in Fig. 1 and Fig. 2, respectively. 
Table 2

Genome-wide gene-environment interaction study results between smoking during pregnancy and short sleep in female offspring

\begin{tabular}{|c|c|c|c|c|}
\hline CHR & SNP & GENE & $T$ & P-value \\
\hline 12 & rs78408032 & ANKLE2 & -5.68 & $1.33 \times 10^{-8}$ \\
\hline 12 & rs75348182 & ANKLE2 & -5.06 & $4.17 \times 10^{-7}$ \\
\hline 2 & rs76688230 & MYT1L & 4.92 & $8.85 \times 10^{-7}$ \\
\hline 2 & rs76840858 & MYT1L & 4.88 & $1.08 \times 10^{-6}$ \\
\hline 7 & rs212421 & ATXN7L1 & -4.84 & $1.30 \times 10^{-6}$ \\
\hline 10 & rs72767679 & CAMK1D & -4.83 & $1.39 \times 10^{-6}$ \\
\hline 8 & rs4732681 & & 4.78 & $1.71 \times 10^{-6}$ \\
\hline 2 & rs10206895 & SERPINE2 & -4.78 & $1.78 \times 10^{-6}$ \\
\hline 2 & rs17392403 & & -4.60 & $4.30 \times 10^{-6}$ \\
\hline 2 & rs12992602 & & -4.58 & $4.56 \times 10^{-6}$ \\
\hline 8 & rs80216884 & & 4.58 & $4.70 \times 10^{-6}$ \\
\hline 8 & rs75079746 & & 4.57 & $4.87 \times 10^{-6}$ \\
\hline 8 & rs6996030 & & 4.56 & $5.15 \times 10^{-6}$ \\
\hline 1 & rs757460970 & & -4.55 & $5.26 \times 10^{-6}$ \\
\hline 2 & rs6436456 & SERPINE2 & -4.54 & $5.63 \times 10^{-6}$ \\
\hline 2 & rs6436457 & SERPINE2 & -4.51 & $6.51 \times 10^{-6}$ \\
\hline 2 & rs4667836 & GALNT3 & 4.50 & $6.68 \times 10^{-6}$ \\
\hline 6 & rs72825589 & ATXN1 & 4.50 & $6.93 \times 10^{-6}$ \\
\hline 2 & rs7607919 & MYT1L & 4.47 & $7.78 \times 10^{-6}$ \\
\hline 14 & rs1969795 & & -4.47 & $7.80 \times 10^{-6}$ \\
\hline 2 & rs10184062 & SERPINE2 & -4.47 & $7.97 \times 10^{-6}$ \\
\hline
\end{tabular}

Note: CHR, chromosome 


\begin{tabular}{|c|c|c|c|c|}
\hline CHR & SNP & GENE & $\mathrm{T}$ & $P$-value \\
\hline 5 & rs17474643 & & 4.46 & $8.29 \times 10^{-6}$ \\
\hline 5 & rs17067202 & & 4.45 & $8.41 \times 10^{-6}$ \\
\hline 2 & rs 6436451 & SERPINE2 & -4.45 & $8.52 \times 10^{-6}$ \\
\hline 8 & rs77935376 & & 4.45 & $8.78 \times 10^{-6}$ \\
\hline 2 & rs2118409 & SERPINE2 & -4.44 & $8.94 \times 10^{-6}$ \\
\hline 2 & rs13411332 & SERPINE2 & -4.44 & $8.97 \times 10^{-6}$ \\
\hline 8 & rs58703445 & & 4.44 & $9.06 \times 10^{-6}$ \\
\hline 14 & rs7159711 & & -4.44 & $9.09 \times 10^{-6}$ \\
\hline 2 & rs1530021 & SERPINE2 & -4.44 & $9.10 \times 10^{-6}$ \\
\hline 2 & rs6436453 & SERPINE2 & -4.44 & $9.12 \times 10^{-6}$ \\
\hline 2 & rs1530020 & SERPINE2 & -4.44 & $9.15 \times 10^{-6}$ \\
\hline 2 & rs3948261 & SERPINE2 & -4.43 & $9.31 \times 10^{-6}$ \\
\hline 2 & rs7584131 & SERPINE2 & -4.43 & $9.49 \times 10^{-6}$ \\
\hline 2 & rs7584056 & SERPINE2 & -4.43 & $9.54 \times 10^{-6}$ \\
\hline 8 & rs79164849 & & 4.43 & $9.60 \times 10^{-6}$ \\
\hline 2 & rs6436458 & SERPINE2 & -4.42 & $9.73 \times 10^{-6}$ \\
\hline 2 & rs6436454 & SERPINE2 & -4.42 & $9.88 \times 10^{-6}$ \\
\hline
\end{tabular}

\section{Discussion}

In the present study, to explore the potential influence of maternal smoking during pregnancy on sleep duration in female offspring, we conducted an observation study and GEWIS in UK Biobank cohort. Our results showed that maternal smoking during pregnancy can effect sleep duration in offspring, which were consistent with the previous study $[16,23]$.

We found that ankyrin repeat and LEM domain containing 2 (ANKLE2) achieved significant threshold in the GWGEIS for short sleep duration in females. ANKLE2 encodes a member of the LEM family of inner nuclear membrane proteins. The encoded protein functions as a mitotic regulator through postmitotic 
formation of the nuclear envelope. Previous research suggested that $A N K L E 2$ regulates $\mathrm{C}$. elegans nuclear envelope reassembly through barrier-to-autointegration factor (BAF) dehosphorylation [24]. Mutations in this gene cause BAF hyperphosphorylation, which is an important DNA-binding protein that interacts with a unique structural motif called LAP2-Emerin-MAN1 (LEM) domain to establish a connection between chromatin and a family of proteins containing LEM domains [25]. Now there is striking evidence that decreased number of glial cells and neuronal within the brain can lead to microcephaly [26, 27], sleep is associated with decreased neurons [28]. And more recently studies have shown that $A N K L E 2$ gene associated with Congenital microcephaly [29, 30], and it has been proved that genetic mutations can cause short sleep phenotype. Based on our study results, ANKLE2 may contribute to the dysfunction of female sleep regulation.

Myelin transcripyion factor 1 like (MYT1L) is another identified candidate gene, which encodes a member of the zinc finger superfamily of transcription factors. The encoded protein is part of a novel class of cystein-cystein-histidine-cystein zinc finger proteins that function in the animal central nervous system. It associated with neuronal cells and via indirect methods exerts its pro-neuronal function [31]. MYT1L only expresses in the human brain, and plays a critical role in the developing central nervous system, which has been related to schizophrenia, intellectual disability, autism [32]. Our results suggest that MYT1L can be considered as a potential gene affecting sleep, consistent with previous study [33]. Enlarging the comparative analysis of $M Y T 1 L$ and other diseases, we found that several diseases regulated by $M Y T 1 L$ are related to sleep. For example, fibromyalgia associated with sleep disturbance and gene MYT1L is candidate gene fibromyalgia [33,34]. Serpin family E member 2 (SERPINE2) is a member of the serpin family, a group of proteins that inhibit serine proteases. It is a susceptibility gene for chronic obstructive pulmonary disease and human tumors $[35,36]$. It is well known that many chronic diseases are associated with sleep disorders, studies shown a significant association between short-term sleep and chronic obstructive pulmonary disease in female[37].

Until now, the biological mechanism of maternal smoking affecting offspring development remains largely known, but established studies could enhance our understanding of the relationship between maternal smoking during pregnancy and sleep duration in female offspring. On the one hand, smoking during pregnancy can have a long-term effect on offspring through hypothalamic-pituitary-adrenal axis $[38,39]$, and effects developing brain's acetycholine neurotransmitter systems [40,41]. On the other hand, smoking during pregnancy might affect early morphological changes of the placenta, resulting in a decreased surface area and volume of fetal capillaries [42, 43], inducing smaller head circumference at birth, and the identified gene ANKLE2 in our further confirmed this in a certain degree. These mechanisms may partly explain the effect of maternal smoking during pregnancy on the offspring's sleep duration.

With the development of science and technology in recent years, it has been commonly known that many diseases are affected by both the environment and genes. Own to the large sample and the rigorous design of UK Biobank, our results are credible and representative. Nevertheless, several limitations should be noticed. Firstly, though we could detect the influence of maternal smoking during pregnancy on offspring sleep duration, the information of indirect exposure to smoking is missing, such as, second- 
hand smoke. Secondly, the data of maternal smoking during pregnancy were driven from online questionnaire surveys, retrospective measurements and which may increase the possibility of recall bias and measurement. In addition, UK Biobank subjects were European whites, it should be cautious when popularize current studies results to other-race.

In summary, our results suggested effect that maternal smoking during pregnancy has effects on sleep duration in offspring, especially on short-term sleep, which may provide an essential information with the pregnancy health, and highlight the detrimental effect of smoking in health from new perspective.

\section{Declarations}

\section{Acknowledgements}

$\mathrm{JZ}$ and FZ conceived and designed the study; JZ and PM wrote the manuscript; All authors collected the data and $C P$, JY carried out the statistical analyses; $C L, H Z, Y C, Z Z, S C, L L, X Y, Y Y$, and YJ made preparations for the manuscript at first. All authors reviewed and approved the final manuscript.

\section{Ethics approval and consent to participate}

Not applicable

\section{Consent for publication}

Not applicable

\section{Availability of data and materials}

Data were driven from the UK Biobank (UKB) https://biobank.ctsu.ox.ac.uk/

\section{Competing interest}

All authors report no biomedical financial interests or potential conflicts of interest.

\section{Funding}

This study is supported by the National Natural Scientific Foundation of China (81922059).

\section{Author information}

\section{Affiliations}

School of Public Health, Health Science Center, Xi'an Jiaotong University 


\section{Contributions}

JZ and FZ conceived and designed the study; JZ and PM wrote the manuscript; All authors collected the data and CP, JY carried out the statistical analyses; CL, HZ, YC, ZZ, SC, LL, XY, YY, and YJ made preparations for the manuscript at first.

\section{Corresponding author}

Correspondence to Feng Zhang

\section{References}

1. Zhou L, Yu K, Yang L, Wang H, Xiao Y, Qiu G, Liu X, Yuan Y, Bai Y, Li X, et al. Sleep duration, midday napping, and sleep quality and incident stroke: The Dongfeng-Tongji cohort. Neurology. 2020;94(4):e345-56.

2. Lu C, Sun H, Huang J, Yin S, Hou W, Zhang J, Wang Y, Xu Y, Xu H. Long-Term Sleep Duration as a Risk Factor for Breast Cancer: Evidence from a Systematic Review and Dose-Response MetaAnalysis. Biomed Res Int. 2017;2017:4845059.

3. Killgore WD. Effects of sleep deprivation on cognition. Prog Brain Res. 2010;185:105-29.

4. Palma JA, Urrestarazu E, Iriarte J. Sleep loss as risk factor for neurologic disorders: a review. Sleep Med. 2013;14(3):229-36.

5. Raymann RJ, Swaab DF, Van Someren EJ. Cutaneous warming promotes sleep onset. Am J Physiol Regul Integr Comp Physiol. 2005;288(6):R1589-97.

6. Partinen M, Kaprio J, Koskenvuo M, Putkonen P, Langinvainio H. Genetic and environmental determination of human sleep. Sleep. 1983;6(3):179-85.

7. Dauvilliers Y, Maret S, Tafti M. Genetics of normal and pathological sleep in humans. Sleep Med Rev. 2005;9(2):91-100.

8. Khlghatyan J, Evstratova A, Bozoyan L, Chamberland S, Chatterjee D, Marakhovskaia A, Soares Silva T, Toth K, Mongrain V, Beaulieu JM. Fxr1 regulates sleep and synaptic homeostasis. Embo j. 2020;39(21):e103864.

9. Hirano A, Hsu PK, Zhang L, Xing L, McMahon T, Yamazaki M, Ptáček LJ, Fu YH. DEC2 modulates orexin expression and regulates sleep. Proc Natl Acad Sci U S A. 2018;115(13):3434-9.

10. Phillips DH. Smoking-related DNA and protein adducts in human tissues. Carcinogenesis. 2002;23(12):1979-2004.

11. WHO. urges more countries to require large, graphic health warnings on tobacco packaging: the WHO report on the global tobacco epidemic, 2011 examines anti-tobacco mass-media campaigns. Cent Eur J Public Health. 2011;19(3):133, 151. 
12. de la Chica RA, Ribas I, Giraldo J, Egozcue J, Fuster C. Chromosomal instability in amniocytes from fetuses of mothers who smoke. Jama. 2005;293(10):1212-22.

13. Parameshwaran K, Buabeid MA, Karuppagounder SS, Uthayathas S, Thiruchelvam K, Shonesy B, Dityatev A, Escobar MC, Dhanasekaran M, Suppiramaniam V. Developmental nicotine exposure induced alterations in behavior and glutamate receptor function in hippocampus. Cell Mol Life Sci. 2012;69(5):829-41.

14. El Marroun $H$, Schmidt MN, Franken IH, Jaddoe VW, Hofman A, van der Lugt A, Verhulst FC, Tiemeier $\mathrm{H}$, White T. Prenatal tobacco exposure and brain morphology: a prospective study in young children. Neuropsychopharmacology. 2014;39(4):792-800.

15. Boakye D, Wyse CA, Morales-Celis CA, Biello SM, Bailey MES, Dare S, Ward J, Gill JMR, Pell JP, Mackay DF. Tobacco exposure and sleep disturbance in 498208 UK Biobank participants. J Public Health (Oxf). 2018;40(3):517-26.

16. O'Callaghan F, O'Callaghan M, Scott JG, Najman J, Al Mamun A. Effect of maternal smoking in pregnancy and childhood on child and adolescent sleep outcomes to 21 years: a birth cohort study. BMC Pediatr. 2019;19(1):70.

17. Voigt M, Hermanussen M, Wittwer-Backofen U, Fusch C, Hesse V. Sex-specific differences in birth weight due to maternal smoking during pregnancy. Eur J Pediatr. 2006;165(11):757-61.

18. Sudlow C, Gallacher J, Allen N, Beral V, Burton P, Danesh J, Downey P, Elliott P, Green J, Landray M, et al. UK biobank: an open access resource for identifying the causes of a wide range of complex diseases of middle and old age. PLoS Med. 2015;12(3):e1001779.

19. Fan M, Sun D, Zhou T, Heianza Y, Lv J, Li L, Qi L. Sleep patterns, genetic susceptibility, and incident cardiovascular disease: a prospective study of 385292 UK biobank participants. Eur Heart J. 2020;41(11):1182-9.

20. Bycroft C, Freeman C, Petkova D, Band G, Elliott LT, Sharp K, Motyer A, Vukcevic D, Delaneau O, O'Connell J, et al. The UK Biobank resource with deep phenotyping and genomic data. Nature. 2018;562(7726):203-9.

21. Chang CC, Chow CC, Tellier LC, Vattikuti S, Purcell SM, Lee JJ. Second-generation PLINK: rising to the challenge of larger and richer datasets. Gigascience. 2015;4:7.

22. Kraft P, Yen YC, Stram DO, Morrison J, Gauderman WJ. Exploiting gene-environment interaction to detect genetic associations. Hum Hered. 2007;63(2):111-9.

23. Stéphan-Blanchard E, Telliez F, Léké A, Djeddi D, Bach V, Libert JP, Chardon K. The influence of in utero exposure to smoking on sleep patterns in preterm neonates. Sleep. 2008;31(12):1683-9.

24. Asencio C, Davidson IF, Santarella-Mellwig R, Ly-Hartig TB, Mall M, Wallenfang MR, Mattaj IW, Gorjánácz M. Coordination of kinase and phosphatase activities by Lem4 enables nuclear envelope reassembly during mitosis. Cell. 2012;150(1):122-35.

25. Jamin A, Wiebe MS. Barrier to Autointegration Factor (BANF1): interwoven roles in nuclear structure, genome integrity, innate immunity, stress responses and progeria. Curr Opin Cell Biol. 2015;34:61-8. 
26. Naveed M, Kazmi SK, Amin M, Asif Z, Islam U, Shahid K, Tehreem S. Comprehensive review on the molecular genetics of autosomal recessive primary microcephaly (MCPH). Genet Res (Camb). 2018;100:e7.

27. Gilmore EC, Walsh CA. Genetic causes of microcephaly and lessons for neuronal development. Wiley Interdiscip Rev Dev Biol. 2013;2(4):461-78.

28. Steullet P, Cabungcal JH, Bukhari SA, Ardelt MI, Pantazopoulos H, Hamati F, Salt TE, Cuenod M, Do $\mathrm{KQ}$, Berretta S. The thalamic reticular nucleus in schizophrenia and bipolar disorder: role of parvalbumin-expressing neuron networks and oxidative stress. Mol Psychiatry. 2018;23(10):205765.

29. Link N, Chung H, Jolly A, Withers M, Tepe B, Arenkiel BR, Shah PS, Krogan NJ, Aydin H, Geckinli BB, et al. Mutations in ANKLE2, a ZIKA Virus Target, Disrupt an Asymmetric Cell Division Pathway in Drosophila Neuroblasts to Cause Microcephaly. Dev Cell. 2019;51(6):713-29.e716.

30. Shaheen R, Maddirevula S, Ewida N, Alsahli S, Abdel-Salam GMH, Zaki MS, Tala SA, Alhashem A, Softah A, Al-Owain M, et al. Genomic and phenotypic delineation of congenital microcephaly. Genet Med. 2019;21(3):545-52.

31. Mall M, Kareta MS, Chanda S, Ahlenius H, Perotti N, Zhou B, Grieder SD, Ge X, Drake S, Euong Ang C, et al. Myt1l safeguards neuronal identity by actively repressing many non-neuronal fates. Nature. 2017;544(7649):245-9.

32. Shi Y, Shao Q, Li Z, Gonzalez GA, Lu F, Wang D, Pu Y, Huang A, Zhao C, He C, et al. Myt1L Promotes Differentiation of Oligodendrocyte Precursor Cells and is Necessary for Remyelination After Lysolecithin-Induced Demyelination. Neurosci Bull. 2018;34(2):247-60.

33. D'Agnelli S, Arendt-Nielsen L, Gerra MC, Zatorri K, Boggiani L, Baciarello M, Bignami E. Fibromyalgia: Genetics and epigenetics insights may provide the basis for the development of diagnostic biomarkers. Mol Pain. 2019;15:1744806918819944.

34. Keskindag B, Karaaziz M. The association between pain and sleep in fibromyalgia. Saudi Med J. 2017;38(5):465-75.

35. An L, Yang T, Zhang Y, Lin Y, Zhang H, Jiao X, Hua L, Dai H, Wang C. Association of SERPINE2 gene with the risk of chronic obstructive pulmonary disease and spirometric phenotypes in northern Han Chinese population. Mol Biol Rep. 2012;39(2):1427-33.

36. Yang $Y, X i n X, F u X, X u$ D. Expression pattern of human SERPINE2 in a variety of human tumors. Oncol Lett. 2018;15(4):4523-30.

37. Lu C, Liao B, Nie J, Wang W, Wang Y. The association between sleep duration and chronic diseases: a population-based cross-sectional study. Sleep Med. 2020;73:217-22.

38. Haslinger $\mathrm{C}$, Bamert $\mathrm{H}$, Rauh M, Burkhardt T, Schäffer L. Effect of maternal smoking on stress physiology in healthy neonates. J Perinatol. 2018;38(2):132-6.

39. van Dalfsen JH, Markus CR. The influence of sleep on human hypothalamic-pituitary-adrenal (HPA) axis reactivity: A systematic review. Sleep Med Rev. 2018;39:187-94. 
40. Garcia-Rill E, Buchanan R, McKeon K, Skinner RD, Wallace T. Smoking during pregnancy: postnatal effects on arousal and attentional brain systems. Neurotoxicology. 2007;28(5):915-23.

41. Saint-Mleux B, Eggermann E, Bisetti A, Bayer L, Machard D, Jones BE, Mühlethaler M, Serafin M. Nicotinic enhancement of the noradrenergic inhibition of sleep-promoting neurons in the ventrolateral preoptic area. J Neurosci. 2004;24(1):63-7.

42. Jauniaux E, Burton GJ. The effect of smoking in pregnancy on early placental morphology. Obstet Gynecol. 1992;79(5 (Pt 1)):645-8.

43. Bush PG, Mayhew TM, Abramovich DR, Aggett PJ, Burke MD, Page KR. A quantitative study on the effects of maternal smoking on placental morphology and cadmium concentration. Placenta. 2000;21(2-3):247-56.

\section{Figures}




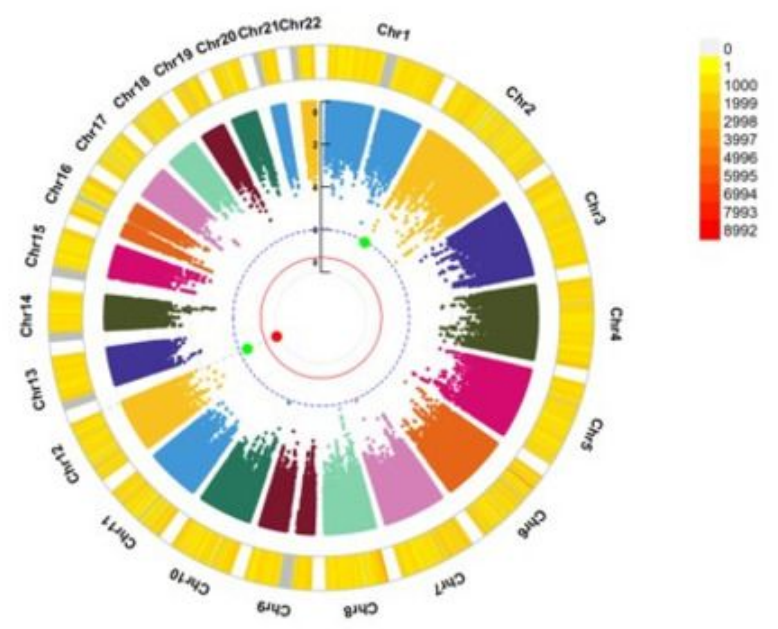

(b)

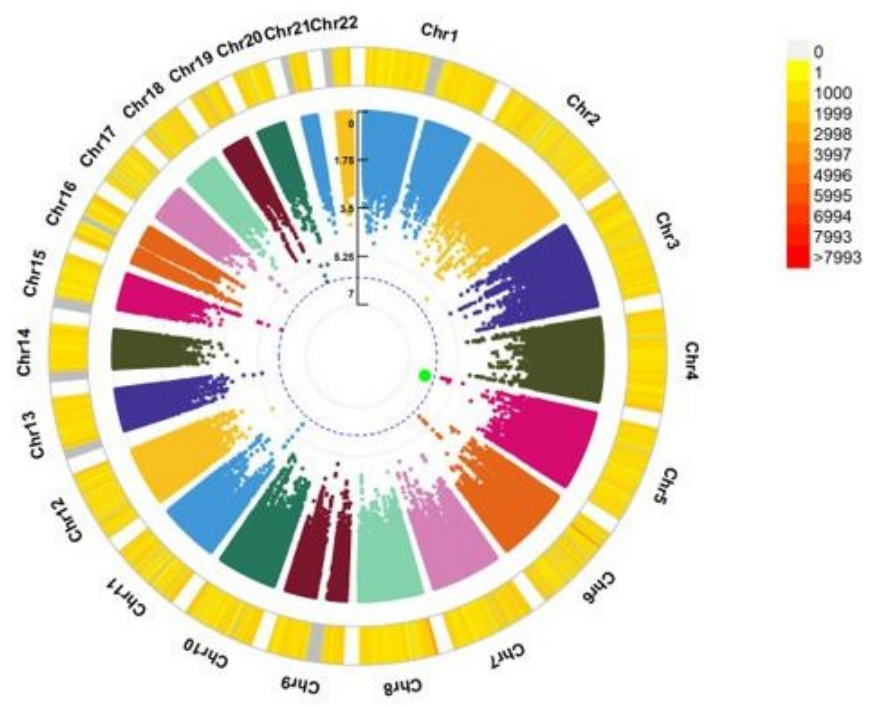

Figure 1

(a-b) Genomic regions interacting with maternal smoking during pregnancy for short sleep (a) and long sleep (b) duration in female offspring Note. From the center, the first circle depicts the -log10 p-values of each variant due to double exposure. The second circle shows chromosome density. The plots were generated using the "CMplot" R script (https://github.com/YinLiLin/R-CMplot). 


\section{Supplementary Files}

This is a list of supplementary files associated with this preprint. Click to download.

- SupplementaryTableS1.docx 